\title{
I-EMO: An Interactive Evolutionary Multi-objective Optimization Tool
}

\author{
Kalyanmoy Deb and Shamik Chaudhuri \\ Kanpur Genetic Algorithms Laboratory (KanGAL), \\ Indian Institute of Technology, Kanpur, \\ Kanpur, PIN 208016, India \\ $\{$ deb, shamik\}@iitk.ac.in, \\ http://www.iitk.ac.in/kangal
}

\begin{abstract}
With the advent of efficient techniques for multi-objective evolutionary optimization (EMO), real-world search and optimization problems are being increasingly solved for multiple conflicting objectives. During the past decade, most emphasis has been spent on finding the complete Pareto-optimal set, although EMO researchers were always aware of the importance of procedures which would help choose one particular solution from the Pareto-optimal set for implementation. This is also one of the main issues on which the classical and EMO philosophies are divided on. In this paper, we address this long-standing issue and suggest an interactive EMO procedure which, for the first time, will involve a decision-maker in the evolutionary optimization process and help choose a single solution at the end. This study is the culmination of many year's of research on EMO and would hopefully encourage both practitioners and researchers to pay more attention in viewing the multi-objective optimization as an aggregate task of optimization and decision-making.
\end{abstract}

\section{Introduction}

In the modern-day design and decision-making process, optimization plays a major role. Till now, most studies concentrated in finding the global optimum in a problem. However, with the advent of proper statistical tools designers and decision-makers are interested in introducing uncertainties and reliability-based concepts in the optimization process. These concepts are introduced simply because the real-world problem is full of such uncertainties and often the global optimum of an idealized deterministic optimization problem is not quite reliable.

In the context of multi-objective optimization and decision-making, there are additional problems of finding a set of optimal solutions and then making a scientific decision in choosing a particular solution. Often, such a decision making process is subjective and only when adequate information about the nature of the optimal solutions is provided to the Decision Maker (DM), a proper and reliable decision-making can be achieved. In this direction, the classical multiobjective literature provides a number of studies in which interactive methods were suggested. The sheer number of different methods also tells that none of 
these methods is widely accepted as a generic procedure. One common matter with all these methods is that they demand a lot of problem information from the DM without providing any idea of the nature of the resulting Pareto-optimal front. Thus, in most cases, the DM, while using these methods, rely on guesswork.

On the other hand, the research focus in evolutionary multi-objective optimization (EMO), was to find the nature and shape of the Pareto-optimal front by using a number of well-distributed solutions. In their history of about 10 years, the researchers did not seem to address the important issue of selecting one solution from the front. In this paper, for the first time, we suggest an interactive procedure (I-EMO), which takes the positive aspects of each field and attempts to suggest a procedure which can iteratively and interactively with a DM find the complete front and help focus to a single preferred solution. Based on the idea, we also provide some snapshots from a software which we are currently developing at Kanpur GA laboratory.

\section{Related Works}

There exists different interactive multi-objective optimization methods in the literature. But most of them are developed based on the classical optimization methods. Some of the most popular ones are Interactive Surrogate Worth Tradeoff (ISWT) method [3], Reference Point method [11, the NIMBUS approach [9] etc. Each method is different from each other, but uses a single solution in each iteration. Based on particular multi-objective optimization procedure, a guess solution is modified to another solution iteratively and by gathering some information from a DM. Since a single solution is used in an iteration, the DM often has a local information (such as a local trade-off or search direction) and cannot make a decision using a more global picture of the true Pareto-optimal front. However, in the context of EMO, there does not exist many interactive studies. Tan et al. developed a GUI-based MOEA toolbox for multi-objective optimization [10]. The toolbox is designed with some classical decision-making aides, such as goal and priority settings. But a clear procedure of arriving at a single preferred solution is not present in the toolbox. Fonseca and Fleming [7] devised a GUI-based procedure which allowed a target value to be set for each objective and the effect of such a setting on the trade-off among different objectives was demonstrated. The procedure can be used to choose a preferred solution by visually comparing different solutions obtained using an EMO. However, the procedure did not provide any mean of relating the obtained solutions (closeness and location on the front) with the true Pareto-optimal front.

\section{Interactive Evolutionary Multi-objective Optimization}

In the proposed interactive EMO procedure, we attempt to put together some recent salient research results of EMO (described below) to constitute an interactive multi-criterion decision-making procedure: 
1. An EMO is capable of finding the entire or a partial Pareto-optimal set, as desired 42$]$.

2. An EMO is capable of finding the knee solutions from the Pareto-optimal set [1].

3. An EMO is capable of finding the robust solutions [5].

4. A local search starting from the EMO-obtained solutions has better convergence properties 6 .

5. An EMO is capable of handling nonlinear constraints with extra additional parameter [4].

Further, in order to make a reliable decision, we ensure a verification procedure based on available multi-objective optimization techniques so that the decisionmaker is satisfied and confident in deciding every step. The following classical principles can be used for this purpose and also for assisting in choosing a specific solution:

1. The extreme Pareto-optimal solutions can be verified by finding the individual optimum of each objective function (with constraints) [9].

2. Multiple applications of $\epsilon$-constraint method can generate a set of Paretooptimal solutions [9].

3. Interactive multi-objective methods, involving Tchebyshev methods or surrogate trade-off information [9] can be used to help choose a particular solution from the Pareto-optimal set.

In the following, we suggest an interactive-EMO procedure. The parameters to be supplied by the decision-maker (DM) are mentioned in parenthesis.

Step 1: Apply an EMO to obtain a non-dominated front close to the true Pareto-optimal front with following options:

1.1 Compute the complete front (DM: no parameter)

1.2 Compute a partial front (DM: limiting trade-off values)

1.3 Compute knee solutions only (DM: limiting angle)

1.4 Compute the robust Pareto-frontier using one of the following two strategies:

1.4.1 Using mean objective values (DM: neighborhood size)

1.4.2 Constrained approach (DM: neighborhood size and limiting robustness parameter)

Step 2: Improve the obtained non-dominated front using other optimization methods:

2.1 Extreme solution improvement: Single-objective optimization of each objective

2.2 Intermediate solution improvement: Local search from solutions chosen as follows:

2.2.1 Automated selection of solutions from non-dominated front: Clustering (DM: number of solutions)

2.2.2 User-defined solutions:

2.2.2.1 Weighted-sum approach (DM: weight vectors) 


\subsubsection{Utility function based approach (DM: utility functions)}

2.2.2.3 Tchebyshev function approach (DM: ideal points and $L_{p}$ norm) 2.3 Construct the modified front

Step 3: Final improvement (if any) with a classical approach

$3.1 \epsilon$-constraint method (one or multi-objective) (DM: $\epsilon$-vector)

3.2 Construct the final front

Step 4: User chooses a particular region of interest (single or multiply-connected) for focussed search using the above procedure

Step 5: Until satisfied, go to Step 1, else declare the chosen solution(s)

As clear from the above procedure, I-EMO proposes to first find the complete or a portion of the Pareto-optimal front in a problem. If desired, the DM can only consider the knee solutions or a robust frontier to start with. Thereafter, the obtained front can be improved by (i) finding individual optimum of each objective and (ii) performing a local search from a preferred set of intermediate solutions from the front. Finally, the classical $\epsilon$-constraint method can be independently used to have a better confidence on the obtained front. Such a combination of a search based on optimization basics and a verification procedure will enable a DM to make his/her decisions more reliably and with confidence in an iterative manner till a single solution is identified. Another important matter is that the DM gets to analyze the true trade-off frontier before making any decision, a matter which makes this procedure different from other existing procedures.

\subsection{In the Making of the I-EMO Software}

At present to carry out such a rigorous interactive study, there exists no software. For an adequate user interaction we need a software with a powerful GUI and a good file-support facility. To this date, a major part of the above activities are coded and we give a flavor of the software using a few screen snap-shots.

In the first screen, the DM needs to define the main parameters of I-EMO, i.e. number of variables, objective functions and constraints to describe the problem. Each parameter is associated with an Edit button. Using this facility, further details of a parameter can be provided. An example is shown for the objective function button in Figure 1. In that window the DM can code his/her objective function(s) using the C-language syntax. There is a GA parameter window for providing population size, maximum generation number, crossover and mutation probabilities etc. Figure 2 shows the obtained non-dominated front for an example problem solved using NSGA-II. On this window, several menu buttons are available: Point Menu, Utility Menu, $\epsilon$-constraint Menu and Individual function Optimization Menu. These menus can be used to choose a set of preferred solutions from which a local search can be performed. This is done to investigate if the solutions can be improved any further. The pop-up window for 'Utility' menu is shown in Figure 3. In the first option, the DM can give his priority of objective functions in the corresponding text-boxes and press the 'Find' button to get the best match on the non-dominated front. Similarly, there exists other 


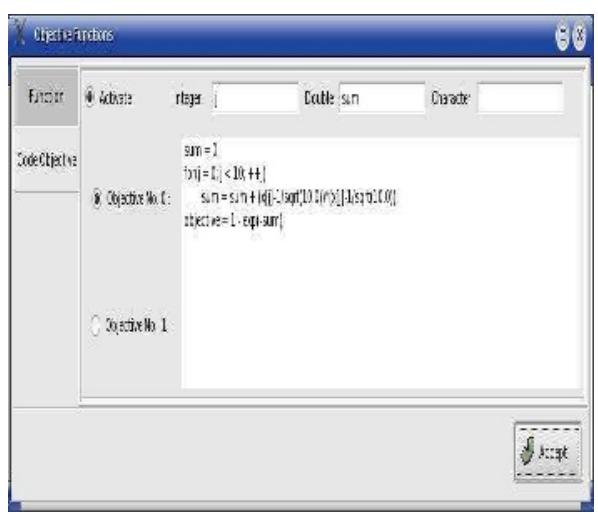

Fig. 1. Window for coding objective functions

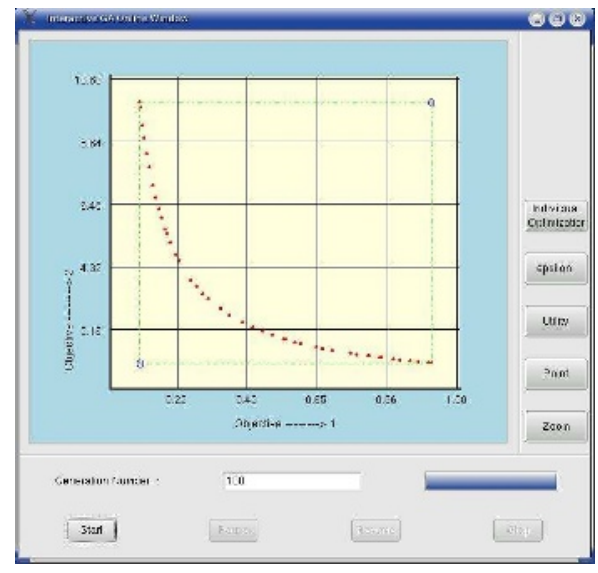

Fig. 2. Plot window for two-objective problem

buttons for providing information via other methods. For example, for Tcebycheff's weight metric method, the DM can pick the reference point on the plot window and define the norm and weight-vector in the menu and ask for the best match. The software also allows the DM to find the individual optimum of each objective function. Then single objective GA is run with this modification and the data is shown in the same window.

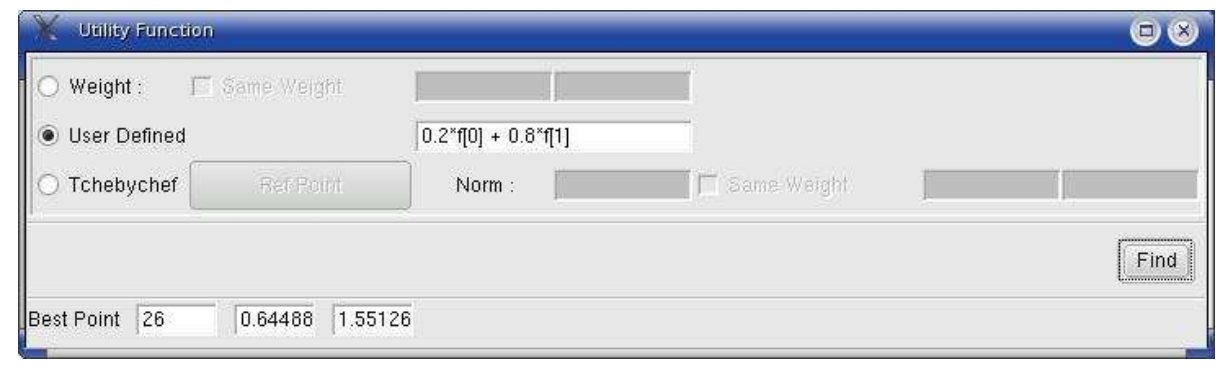

Fig. 3. Utility function window

Visualizing the Pareto-optimal front is an important part of an interactive EMO. However, due to the limitations in visualizing more than three objectives with current practices (albeit some recent suggestions [9]8]), our current implementation is restricted to three objectives only, although any number of objectives can be handled but visualization can be achieved by choosing any two or three objectives one at a time. 


\section{Conclusion}

In this paper, we, for the first time, have proposed a truly interactive procedure for solving multi-objective optimization and decision-making problems. In order to arrive at the procedure, we have used most salient research results from classical and evolutionary multi-objective optimization literatures in a synergistic manner. The procedure not only finds the true Pareto-optimal front and then helps the DM to choose a particular solution, the procedure includes checks and balances at various steps so that the DM is more confident than ever in arriving at a particular solution. Till now, no such combined (classical and EMO) procedure is available for this task. With the ground-breaking research and application studies using EMO, it is now time for researchers to think and develop such interactive methods which will make the multi-objective optimization studies a real practical meaning and useful.

\section{References}

1. J. Branke, K. Deb, H. Dierolf, and M. Osswald. Finding Knees in multi-objective Optimization. Technical Report 2004010, Indian Institute of Technology, Kanpur: Kanpur Genetic Algorithms Laboratory (KanGAL), 2004.

2. J. Branke, T. Kaußler, and H. Schmeck. Guidance in Evolutionary Multi-objective Optimization. Advances in Engineering Software, 32:499-507, 2001.

3. V. Chankong and Y. Y. Haimes. Multiobjective Decision Making Theory and Methodology. New york: North-Holland, first edition edition, 1983.

4. K. Deb. Multi-objective Optimization using Evolutionary Algorithms. Chichester, UK: Wiley, second edition edition, 2001.

5. K. Deb and H. Gupta. Introducing Robustness in Multi-objective Optimization. Technical Report 2004016, Indian Institute of Technology, Kanpur: Kanpur Genetic Algorithms Laboratory (KanGAL), 2004.

6. K. Deb and S. Jain. Running performance metrics for evolutionary multi-objective optimization. In Proceedings of the Fourth Asia-Pacific Conference on Simulated Evolution and Learning (SEAL-02), pages 13-20, 2002.

7. C. M. Fonseca and P. J. Fleming. Multiobjective optimization and multiple constraint handling with evolutionary algorithms-Part II: Application example. IEEE Transactions on Systems, Man, and Cybernetics: Part A: Systems and Humans, 28(1):38-47, 1998.

8. A. V. Lotov, V. A. Bushenkov, and H. K. Kamenev. Interactive Decision Maps. Kluwer, Boston, 2004.

9. K. Miettinen. Nonlinear Multiobjective Optimization. Kluwer's Academic Publisher, Boston, 1999.

10. K. C. Tan, T. H. Lee, D. Khoo, and E. F. Khor. A multiobjective evolutionay algorithm toolbox for computer-aided multiobjective optimization. IEEE Transactions on Systems, Man, and Cybernetics - Part B: Cybernetics, 31(4):537-556, 2001.

11. A.P. Wierzbicki. Reference point approaches. In T. Gal, T. Stewart, and T. Hanne, editors, Multicriteria Decision Making: Advances in MCDM Models, Algorithms, Theory, and Applications, pages 9.1-9.39. Kluwer Academic Publishers, Boston, 1999. 